vantages of the isinglass-plaster, above described, it possesses one other; it is perfectly transparent, so that the surgeon sees in what state of apposition are the edges of the wound, and if a small collection of matter


by merely clipping the plaster; all these advantages of the isinglass-plaster, which are undoubtedly so great as to deserve its general adoption, we have had ample opportunities of witnessing in the surgical practice at the North London Hospital.

Thirdly, MToderate temperature is necessary to adhesion, too high a temperature interfering with the process, as also does a very low degree of temperature, but rather a low temperature is favourable to it.

We have seen in what way superfluous dressings tend to produce the suppurative rather than the adhesive process; there remains, therefore, nothing to be done in order to complete this rather long communication, but to make a remark on bandaging, which, when used too early, not only produces injury by augmenting the temperature, but, when injudiciously applied, by its pressure impedes the return of the blood, and greatly irritates the parts, exciting (by pressure) inflammation and suppuration, and causing absorption of the cellular membrane.* However, a bandage judiciously applied at a later period is frequently very serviceable.

\section{MR. LEWIS'S CURE OF HYDROCELE BY ONE ACUPUNCTURE.}

\section{To the Editor of The LANCET.}

SIR:-In the last number of the Medical Gazette there is a long and uncourteous letter from Mr. Travers, in which he seems to insinuate that he was the discoverer of the new method of treating hydrocele, as detailed by me in THE LANCET of the 7 th of May last, because he had used an acupuncturation-needle in a case of hydrocele, a fortnight prior to the date of my letter. That such may have been the case, I am not in a position to deny, although $I$ must, in justice to myself, declare, that I never heard of Mr. Travers deviating from the ancient routine of practice in the treatment of hydrocele, or any other disease. But lest the public should imagine, like Mr. Travers, that my discovery, and the appearance of the letter in THE LANCET, were synchronous, $I$ can bring forward evidence that the operation had been performed two years before Mr. Travers " conceived" it, as he says. I even spoke of it in public to several medical gentlemen, who will, I have no doubt, vouch for the accuracy of this state. ment. It was not until I had perfected the

* See Professor Cooper's First Lines of the Practice of Surgery, p. 709. operation, and had seen many cases, that I dared to make public the extraordinary fact, that a single puncture, with the point of a fine needle, into an encysted carity, is suf. ficent to cause the absorption of the fluid contained therein. The practice of making many punctures, (Mr. Travers's plan), is not only useless and dangerous, but actually less likely to succeed than a single punc. ture. Of this, the profession in general (I mean general practitioners as well as hos. pital surgeons), will be convinced by ex. perience. If Mr. Travers had read atten. tively my letter, he would have seen that I first introduced the method as a palliative cure, although I have seen cases where a radical cure was effected by the simple operation of puncturation. As the method of puncturation for the radical cure of hy. drocele differs in some respects from the palliative, so soon as I have satisfied my mind on the subject, I shall rejoice, (humble an individual as $\mathrm{I} \mathrm{am}$ ), to contribute my mite towards the advancement of science. I am, Sir, your's truly,
28, Artillery-place West, Bunhill-row, Feb. 14, 1837 .

\section{CLOSURE OF PORT-MORTEM INCISIONS.}

\section{To the Editor of The IAxceT.}

Sir :-Among the numerous ingenions mechanical constructions which have at intervals emanated from the establishments of instrument makers, for the use of the medical practitioner, it is surprising no coll. trivance has been offered, by means of whicl the incisions in port-mortem examinations might be more expeditiously and safely closed. Either so unimportant a contriv. ance has not been deemed of sufficient im. portance for the exercise of skill, or the need for it has not occurred to any mechanic. There is, however, a necessity for additional auxiliaries in this department. The exter. nal parts are placed in situ by a most awk. ward and dangerous sharp-pointed needle, in the use of which, however careful the work. man, occasional punctures result, with, pos. sibly, serious temporary inconvenience, $0 t$, in some instances, a fatal consequence. I would suggest the construction of a pair of mode. rate-sized hand-forceps, somewhat similar in appearance and operation to the shoe. maker's spring punch, except that in lien of the cutter, one of several variously lengtl. ened, sharp, straight needles (of proportion. ate depth to the thickness of the integu. ment) should screw into the upper blade of the folceps, and meet a foramen cacum in its fellow, hollowed so gradually as to prevent the possibility of injuring the apex of the instrument. Thus the requisite number 